\title{
COUPLING A STOCHASTIC APPROXIMATION VERSION OF EM WITH AN MCMC PROCEDURE*
}

\author{
Estelle $\mathrm{KuHN}^{1}$ AND MARC LAVIELle ${ }^{2}$
}

\begin{abstract}
The stochastic approximation version of EM (SAEM) proposed by Delyon et al. (1999) is a powerful alternative to EM when the E-step is intractable. Convergence of SAEM toward a maximum of the observed likelihood is established when the unobserved data are simulated at each iteration under the conditional distribution. We show that this very restrictive assumption can be weakened. Indeed, the results of Benveniste et al. for stochastic approximation with Markovian perturbations are used to establish the convergence of SAEM when it is coupled with a Markov chain Monte-Carlo procedure. This result is very useful for many practical applications. Applications to the convolution model and the change-points model are presented to illustrate the proposed method.
\end{abstract}

Mathematics Subject Classification. 62F10, 62L20, 65C40.

Received May 4, 2003. Revised November 2, 2003.

\section{INTRODUCTION}

A wide class of statistical problems involves observed and unobserved data. We can consider, for example, inverse problems concerning deconvolution, source separation, change-points detection, etc. Linear and nonlinear mixed effects models can also be considered incomplete-data models. Estimation of the parameters of these models is a difficult challenge. In particular, the likelihood of the observations cannot usually be maximized in closed form.

The expectation-maximization (EM) algorithm, proposed by Dempster, Laird and Rubin [6], is a broadly applicable approach for the iterative computation of maximum likelihood estimates, useful in a variety of incomplete-data (or partially-observed-data) statistical problems. The standard incomplete-data scheme considers the observable incomplete data $y \sim g(y ; \theta)$ to result from partial observation of complete data $(y, z) \sim$ $f(y, z ; \theta)$, where $g$ and $f$ are some known density functions. Maximum likelihood estimation of $\theta$ consists in computing the value of $\theta$ that maximizes the observed likelihood $g$.

The E-step of the EM algorithm computes $Q\left(\theta \mid \theta_{k}\right)=\mathrm{E}\left[\log f(y, z ; \theta) \mid y ; \theta_{k}\right]$ and the M-step determines $\theta_{k+1}$ as maximizing $Q\left(\theta \mid \theta_{k}\right)$. Then, the observed-data likelihood sequence $\left(g\left(y ; \theta_{k}\right)\right)$ is nondecreasing along any EM sequence, see [15] for more details. Stochastic versions of EM have been introduced from different perspectives

\footnotetext{
Keywords and phrases. EM algorithm, SAEM algorithm, stochastic approximation, MCMC algorithm, convolution model, change-points model.

* This work was supported by Comity ECOS-Nord, No. V00M03.

1 Université Paris Sud, Bât. 425, 91400 Orsay, France; e-mail: Estelle.Kuhn@math.u-psud.fr

2 Université René Descartes and Université Paris Sud, Bât. 425, 91400 Orsay, France; e-mail: Marc.Lavielle@math.u-psud.fr
} 
to deal with situations where the E-step is infeasible in closed form. Monte-Carlo EM (MCEM) replaces this step by a Monte-Carlo approximation based on a large number of independent simulations of the missing data, see [12]. The SAEM algorithm, proposed by Delyon, Lavielle and Moulines [5], replaces the E-step by stochastic approximation. The $k$-th step of SAEM generates $m(k)$ realizations $z_{k}(j)(1 \leq j \leq m(k))$ from $p\left(z \mid y ; \theta_{k}\right)$ and updates $Q_{k-1}(\theta)$ according to

$$
Q_{k}(\theta)=Q_{k-1}(\theta)+\gamma_{k}\left(\frac{1}{m(k)} \sum_{j=1}^{m(k)} \log f\left(y, z_{k}(j) ; \theta\right)-Q_{k-1}(\theta)\right)
$$

where $\left(\gamma_{k}\right)$ is a sequence of positive step sizes decreasing to 0 . It is possible to select $m(k)=1$ for all $k$ when simulation of $z_{k}$ is heavy. Then the M-step consists in determining $\theta_{k+1}$ which maximizes $Q_{k}(\theta)$. Precise results of convergence of SAEM are presented in [5] in the case where $f(y, z ; \theta)$ belongs to a regular curved exponential family. In this case, SAEM can be written in terms of the complete-data sufficient statistics. This leads to a general Robbins-Monro-type scheme and the almost sure convergence of the sequence $\left(\theta_{k}\right)$ to a local maximum of the likelihood is proved under very general assumptions.

Unfortunately, for most nonlinear models or non-Gaussian models, the unobserved data cannot be simulated exactly under the conditional distribution. A well-known alternative consists in using a Metropolis-Hastings algorithm: introduce a transition probability which has as unique invariant distribution the conditional distribution we want to simulate. In this situation, the assumptions of [5] that ensure the convergence of SAEM are no longer satisfied. The aim of this paper is to show that these assumptions can be weakened such that SAEM still converges when it is coupled with a Markov chain Monte-Carlo procedure. The results of Benveniste et al. [1] for stochastic approximation with Markovian perturbations are used to establish the convergence of this algorithm.

The use of simulated data for estimating parameters is a powerful approach that has become popular in recent years. In [16], Yao defines and studies an online stochastic approximation scheme. In this situation, the number of observations goes to infinity and the sequence of estimates converges to the true value of the parameter. In [7], Gu and Kong propose a stochastic version of a Newton-Raphson algorithm for incompletedata estimation. The algorithm proposed by Gu and Zhu in [8] combines an MCMC procedure with stochastic approximation for spatial models estimation. In these two papers, the information matrix is also estimated by stochastic approximation. In [4], Concordet and Nuñez propose a pseudo simulated maximum likelihood method for estimating the parameters of a nonlinear mixed effects model.

Application of SAEM to the change-points model was proposed in [10] and to the convolution model in [11], but no results of convergence of the algorithm were given. We show that the convergence results obtained for SAEM are very general and apply to these two examples of application. A Monte-Carlo experiment illustrates the performances of the proposed procedure with these two models. We show that the SAEM algorithm can also be used for estimating the asymptotic covariance matrix of the Maximum Likelihood Estimate.

\section{The STOCHASTIC APPROXIMATION VERSION OF EM ALGORITHM}

\subsection{The EM and SAEM algorithms}

Let the complete data $x=(y, z) \in \mathbb{R}^{n+l}$, where $y \in \mathbb{R}^{n}$ is observed and $z \in \mathbb{R}^{l}$ is missing for some $n \in \mathbb{N}$, $l \in \mathbb{N}$. Throughout the sequel, the observed-data vector $y$ is fixed but $z$ is variable. Let $\mu^{\prime}$ be a $\sigma$-finite measure on $\mathbb{R}^{n+l}$ and $\mu$ the restriction of $\mu^{\prime}$ to $\mathbb{R}^{l}$ generated by $x \mapsto z$. Let $\mathcal{P}=\{f(y, z ; \theta), \theta \in \Theta\}$ be a family of probability densities on $\mathbb{R}^{n+l}$ w.r.t. $\mu^{\prime}$, where $\Theta \subset \mathbb{R}^{p}$. We consider in this paper only models for which the complete data 
likelihood $f(y, z ; \theta)$ belongs to the curved exponential family. The incomplete-data likelihood (the likelihood of the observed data $y$ ) is defined by:

$$
g(y ; \theta) \triangleq \int_{\mathbb{R}^{l}} f(y, z ; \theta) \mu(\mathrm{d} z)
$$

Our purpose is to find the value $\widehat{\theta_{g}}$ in $\Theta$ that maximizes the observed likelihood $g$.

In the sequel, $p(z \mid y ; \theta)$ denotes the conditional distribution of the missing data $z$ given the observed data $y$ :

$$
p(z \mid y ; \theta) \triangleq \begin{cases}f(y, z ; \theta) / g(y ; \theta) & \text { if } g(y ; \theta) \neq 0 \\ 0 & \text { if } g(y ; \theta)=0\end{cases}
$$

We shall make the following assumptions on the model, which are the same as these presented in [5]:

- (M1) The parameter space $\Theta$ is an open subset of $\mathbb{R}^{p}$. The complete data likelihood function is given by:

$$
f(y, z ; \theta)=\exp \{-\psi(\theta)+\langle\tilde{S}(y, z), \phi(\theta)\rangle\}
$$

where $\langle\cdot, \cdot\rangle$ denotes the scalar product, $\tilde{S}$ is a Borel function on $\mathbb{R}^{l}$ in the second variable taking its values in an open subset $\mathcal{S}$ of $\mathbb{R}^{m}$. Moreover, the convex hull of $\tilde{S}\left(\mathbb{R}^{l}\right)$ is included in $\mathcal{S}$, and, for all $\theta$ in $\Theta$,

$$
\int_{\mathbb{R}^{l}}|\tilde{S}(y, z)| p(z \mid y ; \theta) \mu(\mathrm{d} z)<\infty
$$

- (M2) Define $L: \mathcal{S} \times \Theta \rightarrow \mathbb{R}$ as:

$$
L(s ; \theta) \triangleq-\psi(\theta)+\langle s, \phi(\theta)\rangle
$$

The functions $\psi$ and $\phi$ are twice continuously differentiable on $\Theta$.

- (M3) The function $\bar{s}: \Theta \rightarrow \mathcal{S}$ defined as

$$
\bar{s}(\theta) \triangleq \int_{\mathbb{R}^{l}} \tilde{S}(y, z) p(z \mid y ; \theta) \mu(\mathrm{d} z)
$$

is continuously differentiable on $\Theta$.

- (M4) The function $l: \Theta \rightarrow \mathbb{R}$ defined as the observed-data log-likelihood

$$
l(\theta) \triangleq \log g(y ; \theta)=\log \int_{\mathbb{R}^{l}} f(y, z ; \theta) \mu(\mathrm{d} z)
$$

is continuously differentiable on $\Theta$ and

$$
\partial_{\theta} \int f(y, z ; \theta) \mu(\mathrm{d} z)=\int \partial_{\theta} f(y, z ; \theta) \mu(\mathrm{d} z) .
$$

- (M5) There exists a function $\widehat{\theta}: \mathcal{S} \rightarrow \Theta$, such that:

$$
\forall s \in \mathcal{S}, \quad \forall \theta \in \Theta, \quad L(s ; \widehat{\theta}(s)) \geq L(s ; \theta)
$$

Moreover, the function $\widehat{\theta}$ is continuously differentiable on $\mathcal{S}$. 
Let us define

$$
Q\left(\theta \mid \theta^{\prime}\right)=\int_{\mathbb{R}^{l}} \log f(y, z ; \theta) p\left(z \mid y ; \theta^{\prime}\right) \mu(\mathrm{d} z) .
$$

In cases where maximization of $\theta \rightarrow Q\left(\theta \mid \theta^{\prime}\right)$ is much simpler than direct maximization of $\theta \rightarrow l(\theta)$, it is useful to apply the EM algorithm which maximizes $l(\theta)$ by iteratively maximizing $Q\left(\theta \mid \theta^{\prime}\right)$. Each iteration of EM can be decomposed into two steps. At iteration $k$, the E-step consists in evaluating $Q\left(\theta \mid \theta_{k}\right)$. Then, the M-step consists in computing $\theta_{k+1}$ by maximizing $Q\left(\theta \mid \theta_{k}\right)$.

Using our notation, the $k$-th iteration of the EM algorithm may be expressed as:

$$
\begin{aligned}
Q\left(\theta \mid \theta_{k}\right) & =L\left(\bar{s}\left(\theta_{k}\right) ; \theta\right) \\
\theta_{k+1} & =T\left(\theta_{k}\right)=\widehat{\theta}\left(\bar{s}\left(\theta_{k}\right)\right) .
\end{aligned}
$$

The convergence of this algorithm is due to the fact that increasing $Q\left(\theta \mid \theta_{k}\right)$ generates an increase in $l\left(\theta_{k}\right)$. This convergence has been studied by many different authors (see $[6,9,15])$ and is ensured, for example, under (M1)-(M5) when the sequence $\left(\theta_{k}\right)_{k \geq 0}$ stays within some compact subset of $\Theta$ (see [5]).

In the SAEM algorithm, the E-step is split into a simulation step (S-step) and a stochastic approximation integration step. At iteration $k$, the S-step consists in generating a realization of the missing data vector $z_{k}$ under the conditional distribution $p\left(\cdot \mid y ; \theta_{k}\right)$ and the integration step in a stochastic averaging procedure:

$$
s_{k}=s_{k-1}+\gamma_{k}\left(\tilde{S}\left(y, z_{k}\right)-s_{k-1}\right),
$$

which is equivalent under the assumption (M1) of a curved exponential family to the general procedure presented in (1). Then, the complete log-likelihood is maximized in the M-step by $\theta_{k+1}=\widehat{\theta}\left(s_{k}\right)$.

Some precise results of convergence of this algorithm were obtained in [5]. First, it is assumed that the random variables $s_{0}, z_{1}, z_{2}, \cdots$ are defined on the same probability space $(\Omega, \mathcal{A}, P)$. We denote $\mathcal{F}=\left\{\mathcal{F}_{k}\right\}_{k \geq 0}$ the increasing family of $\sigma$-algebras generated by the random variables $s_{0}, z_{1}, z_{2}, \cdots, z_{k}$. In addition, we assume that:

- (SAEM1) For all $k$ in $\mathbb{N}, \gamma_{k} \in[0,1], \sum_{k=1}^{\infty} \gamma_{k}=\infty$ and $\sum_{k=1}^{\infty} \gamma_{k}^{2}<\infty$.

- (SAEM2) $l: \Theta \rightarrow \mathbb{R}$ and $\widehat{\theta}: \mathcal{S} \rightarrow \Theta$ are $m$ times differentiable, where $m$ is the integer such that $\mathcal{S}$ is an open subset of $\mathbb{R}^{m}$.

- (SAEM3)

(1) For all positive Borel functions $\Phi$ :

$$
E\left[\Phi\left(z_{k+1}\right) \mid \mathcal{F}_{k}\right]=\int \Phi(z) p\left(z \mid y ; \theta_{k}\right) \mu(\mathrm{d} z) .
$$

(2) For all $\theta \in \Theta, \int\|\tilde{S}(y, z)\|^{2} p(z \mid y ; \theta) \mu(\mathrm{d} z)<\infty$, and the function

$$
\begin{aligned}
\Gamma(\theta) \triangleq \operatorname{Cov}_{\theta}[\tilde{S}(y, z)] \triangleq & \int_{\mathbb{R}^{l}} \tilde{S}(y, z)^{t} \tilde{S}(y, z) p(z \mid y ; \theta) \mu(\mathrm{d} z) \\
& -\left[\int_{\mathbb{R}^{l}} \tilde{S}(y, z) p(z \mid y ; \theta) \mu(\mathrm{d} z)\right]^{t}\left[\int_{\mathbb{R}^{l}} \tilde{S}(y, z) p(z \mid y ; \theta) \mu(\mathrm{d} z)\right]
\end{aligned}
$$

is continuous w.r.t. $\theta$.

It was shown in [5], that the sequence $\left(\theta_{k}\right)_{k \geq 0}$ generated by SAEM converges to a stationary point of the observed likelihood $g$, when (M1)-(M5) and (SAEM1)-(SAEM3) are satisfied, and when the sequence $\left(s_{k}\right)_{k \geq 0}$ takes its values in a compact subset of $\mathcal{S}$.

In many practical situations, it will not be possible to generate the unobserved data $z_{k}$ exactly under the conditional distribution $p\left(\cdot \mid y ; \theta_{k}\right)$. Then, the assumption (SAEM3) will not be satisfied. Nevertheless, we will 
show that (SAEM3) can be replaced by a weaker condition that is satisfied when a Markov chain Monte-Carlo procedure is used in the S-step of SAEM.

\subsection{Coupling the SAEM algorithm with MCMC}

For any $\theta \in \Theta$, assume that the conditional distribution $p(\cdot \mid y ; \theta)$ is the unique limiting distribution of a transition probability $\Pi_{\theta}$. When the missing data $z_{k}$ cannot be generated under $p\left(\cdot \mid y ; \theta_{k}\right)$, we will use the transition kernel $\Pi_{\theta_{k}}$. Then, the $k$-th iteration of the proposed algorithm can be summarized in three steps as follows:

- Simulation: using $z_{k-1}$, generate a realization $z_{k}$ from the transition probability $\Pi_{\theta_{k}}\left(z_{k-1}, \cdot\right)$.

- Stochastic approximation: update $s_{k-1}$ according to (4).

- Maximization: update $\theta_{k}$ according to $\theta_{k+1}=\widehat{\theta}\left(s_{k}\right)$.

Usually, $\Pi_{\theta}$ will be defined as the succession of $M$ iterations of a MCMC procedure, such as the MetropolisHastings algorithm. Then, the S-step of iteration $k$ consists in simulating $z_{k}$ with the transition probability $\Pi_{\theta_{k}}\left(z_{k-1}, \mathrm{~d} z_{k}\right)=P_{\theta_{k}}^{M}\left(z_{k-1}, \mathrm{~d} z_{k}\right)$, where

$$
P_{\theta_{k}}\left(z, \mathrm{~d} z^{\prime}\right)=q_{\theta_{k}}\left(z, z^{\prime}\right) \min \left\{\frac{p\left(z^{\prime} \mid y ; \theta_{k}\right) q_{\theta_{k}}\left(z^{\prime}, z\right)}{p\left(z \mid y ; \theta_{k}\right) q_{\theta_{k}}\left(z, z^{\prime}\right)}, 1\right\} \mathrm{d} z^{\prime}
$$

for $z^{\prime} \neq z$ and $P_{\theta_{k}}(z,\{z\})=1-\int_{z^{\prime} \neq z} P_{\theta_{k}}\left(z, \mathrm{~d} z^{\prime}\right)$, where $q_{\theta}\left(z, z^{\prime}\right)$ is any aperiodic recurrent transition density.

For example, we can use the marginal distribution $\pi$ of $z_{k}$ as a proposal distribution. Then, writing $f(y, z ; \theta)=$ $\pi(z ; \theta) h(y \mid z ; \theta)$, the acceptance probability only depends on the conditional distribution $h$ of the observation $y$ :

$$
P_{\theta_{k}}\left(z, \mathrm{~d} z^{\prime}\right)=\pi\left(z^{\prime} ; \theta_{k}\right) \min \left\{\frac{h\left(y \mid z^{\prime} ; \theta_{k}\right)}{h\left(y \mid z ; \theta_{k}\right)}, 1\right\} \mathrm{d} z^{\prime} .
$$

\subsection{Estimation of the variance of the estimates}

An estimation procedure should generate a point estimate $\widehat{\theta_{g}}$ together with the covariance of the estimate (for instance, to enable construction of confidence sets for the true parameter value). When $g$ is an incomplete data likelihood function sufficiently smooth, asymptotic theory for maximum-likelihood estimation holds and

$$
\sqrt{n}\left(\widehat{\theta_{g}}-\theta^{\star}\right) \rightarrow_{n \rightarrow \infty} \mathcal{N}\left(0, I_{g}\left(\theta^{\star}\right)^{-1}\right)
$$

where $I_{g}\left(\theta^{\star}\right)$ is the observed Fisher information matrix. Thus, an estimate of the asymptotic covariance of $\widehat{\theta_{g}}$ is the inverse of the observed Fisher information matrix $-\partial_{\theta}^{2} l\left(\widehat{\theta_{g}}\right)$.

Using the SAEM algorithm, the observed Fisher information matrix $-\partial_{\theta}^{2} l\left(\widehat{\theta_{g}}\right)$ can be approximated by the sequence $\left(-H_{k}\right)$ defined as follows (see [5] for more details):

$$
\begin{aligned}
\Delta_{k} & =\Delta_{k-1}+\gamma_{k}\left[\partial_{\theta} \log f\left(y, z_{k} ; \theta_{k}\right)-\Delta_{k-1}\right] \\
G_{k} & =G_{k-1}+\gamma_{k}\left(\partial_{\theta}^{2} \log f\left(y, z_{k} ; \theta_{k}\right)+\partial_{\theta} \log f\left(y, z_{k} ; \theta_{k}\right) \partial_{\theta} \log f\left(y, z_{k} ; \theta_{k}\right)^{t}-G_{k-1}\right) \\
H_{k} & =G_{k}-\Delta_{k} \Delta_{k}{ }^{t} .
\end{aligned}
$$

\section{Convergence Result}

We will use some results that work for general Robbins-Monro type stochastic approximation procedures. So if we write the recursion (4) into this form, it becomes:

$$
s_{k}=s_{k-1}+\gamma_{k} h\left(s_{k-1}\right)+\gamma_{k} e_{k}
$$


where $h$ stands for the mean field of the algorithm and $e_{k}$ is a random perturbation. In our case, we obtain the following expressions for the function $h$ and the sequence $\left(e_{k}\right)$ :

$$
\begin{aligned}
h(s) & =E_{\widehat{\theta}(s)}(\tilde{S}(y, z))-s=\bar{s}(\widehat{\theta}(s))-s \\
e_{k} & =\tilde{S}\left(z_{k}\right)-E\left[\tilde{S}\left(z_{k}\right) \mid \mathcal{F}_{k-1}\right]=\tilde{S}\left(z_{k}\right)-\bar{s}\left(\widehat{\theta}\left(s_{k-1}\right)\right)
\end{aligned}
$$

where $\mathrm{E}_{\theta}[\Phi(z)] \triangleq \int \Phi(z) p(z \mid y ; \theta) \mu(\mathrm{d} z)$.

The assumption (SAEM3)1. means that, given $\theta_{0}, \cdots, \theta_{k}$, the random variables $z_{0}, \cdots, z_{k}$ are independent. Coupled with the assumption (SAEM3)2., it is sufficient to prove that the series $\sum \gamma_{k} e_{k}$ converges thanks to the martingale theory. This property plays a key role in the proof of the convergence of the SAEM algorithm in [5]. In this new version of the algorithm, we allow Markovian dependence between $z_{k}$ and $z_{k+1}$ : we suppose that $z_{k+1}$ is obtained from $z_{k}$ due to a Markovian transition depending on $\theta_{k+1}$, so that we will need some technical tools presented in [1] to show that the series $\sum \gamma_{k} e_{k}$ still converges.

As announced above, the assumption (SAEM3) can be weakened. It is assumed that the random variables $s_{0}, z_{1}, z_{2}, \cdots, z_{k}, \cdots$ are defined on the same probability space $(\Omega, \mathcal{A}, P)$. We denote $\mathcal{F}=\left\{\mathcal{F}_{k}\right\}_{k \geq 0}$ the increasing family of $\sigma$-algebras generated by the random variables $s_{0}, z_{1}, z_{2}, \cdots, z_{k}$. Since we introduce the transition probability $\Pi_{\theta}$ in order to approach the conditional distribution, we have to make some assumptions on it:

\section{- (SAEM3')}

1. The chain $\left(z_{k}\right)_{k \geq 0}$ takes its values in a compact subset $\mathcal{E}$ of $\mathbb{R}^{l}$.

2. For any compact subset $V$ of $\Theta$, there exists a real constant $L$ such that for any $\left(\theta, \theta^{\prime}\right)$ in $V^{2}$

$$
\sup _{(x, y) \in \mathcal{E}^{2}}\left|\Pi_{\theta}(x, y)-\Pi_{\theta^{\prime}}(x, y)\right| \leq L\left|\theta-\theta^{\prime}\right| .
$$

3. The transition probability $\Pi_{\theta}$ generates a uniformly ergodic chain whose invariant probability is the conditional distribution $p(\cdot \mid y ; \theta)$ :

$$
\left.\exists K_{\theta} \in \mathbb{R}^{+} \quad \exists \rho_{\theta} \in\right] 0,1\left[\quad \mid \quad \forall z \in \mathcal{E} \quad \forall k \in \mathbb{N} \quad\left\|\Pi_{\theta}^{k}(z, \cdot)-p(\cdot \mid y ; \theta)\right\|_{T V} \leq K_{\theta} \rho_{\theta}^{k},\right.
$$

where $\|\cdot\|_{T V}$ denotes the total variation norm. We suppose also that:

$$
K \triangleq \sup _{\theta} K_{\theta}<+\infty \quad \text { and } \quad \rho \triangleq \sup _{\theta} \rho_{\theta}<1
$$

4. The function $\tilde{S}$ is bounded on $\mathcal{E}$.

We obtain the following convergence result, which is analogous to Theorem 5 of Delyon et al. [5]:

Theorem 1. Assume that assumptions (M1-M5), (SAEM1-SAEM2) and (SAEM3') hold. Assume in addition the assumption $(\mathbf{C})$ : the sequence $\left(s_{k}\right)_{k \geq 0}$ takes its values in a compact subset of $\mathcal{S}$. Then, w.p. 1, $\lim _{k \rightarrow+\infty} d\left(\theta_{k}, \mathcal{L}\right)=0$ where $d(x, A)$ denotes the distance of $x$ to the closed subset $A$ and $\mathcal{L}=\left\{\theta \in \Theta, \partial_{\theta} l(y ; \theta)=\right.$ $0\}$ is the set of stationary points of $l$.

\section{Remarks:}

- For checking assumption (SAEM3')3, it is possible to verify some minorization condition or Doeblin's condition for the transition probability $\Pi_{\theta}$ (see Chap. 16 of [14]). Otherwise, we have to consider each case individually. Consider for example an independent Metropolis-Hastings algorithm: the transition density $q_{\theta}$ mentioned in equation (5) defines an independence sampler:

$$
\forall\left(z, z^{\prime}\right) \in \mathcal{E}^{2} \quad q_{\theta}\left(z, z^{\prime}\right)=q_{\theta}\left(z^{\prime}\right)
$$


Then the uniform ergodicity is ensured if the transition $q_{\theta}$ satisfies the following inequality (see Th. 2.1 in [13]):

$$
\exists \beta \in \mathbb{R}^{+} \quad \mid \quad \forall z \in \mathcal{E} \quad q_{\theta}(z) \geq \beta p(z \mid y ; \theta) .
$$

- In cases where the compactness condition (C) is not checked or is difficult to check, it is possible to stabilize the algorithm by using the method of dynamic bounds proposed by Chen et al. in [3] and already used in this context by Delyon et al. in [5]: if $s_{k}$ is outside a given compact set $\mathcal{K}_{k}$ of $\mathcal{S}$, it is reinitialized in a specific compact set $\mathcal{K}_{0}$.

- It was shown in [5] that, under reasonable conditions, SAEM a.s. avoids traps, i.e., can only converge to a proper local maximizer of the likelihood. This result still holds in our context. Indeed, the paragraph II.3. of [2] about little Markovian perturbations gives some sufficient conditions for avoiding the local minima or saddle points of $l$ :

$$
\liminf \lambda_{\min }\left(E\left[\left(\tilde{S}\left(z_{n+1}\right)-s^{\star}\right)\left(\tilde{S}\left(z_{n+1}\right)-s^{\star}\right)^{t} \mid \mathcal{F}_{n}\right]\right)>0 \text { p.s. on } \Gamma\left(s^{\star}\right)
$$

where $\lambda_{\min }(A)$ denotes the smallest eigenvalue of $A$ and $\Gamma\left(s^{\star}\right)$ is the set of the sequences $\left(s_{k}\right)$ which converge to $s^{\star}$.

Proof of Theorem 1. Theorem 1 is an application of Theorem 2 presented in [5], which gives a general result about convergence of Robbins-Monro type stochastic approximation procedures of the form (11):

Theorem (Th. 2 of Delyon et al. [5]). Assume that:

- (SA0) w.p.1, $\forall k \geq 0, s_{k} \in \mathcal{S}$.

- (SA1) $\left(\gamma_{k}\right)_{k \geq 0}$ is a decreasing sequence of positive number such that $\sum_{k=1}^{\infty} \gamma_{k}=\infty$.

- (SA2) The vector field $h$ is continuous on $\mathcal{S}$ and there exists a continuously differentiable function $V: \mathcal{S} \rightarrow \mathbb{R}$ such that:

1. $\forall s \in \mathcal{S} \quad F(s)=\left\langle\partial_{s} V(s), h(s)\right\rangle \leq 0$.

2. $\operatorname{int}(V(\mathcal{L}))=\emptyset$, where $\mathcal{L} \triangleq\{s \in \mathcal{S}, F(s)=0\}$.

- (SA3) w.p.1, the closure of $\left(\left\{s_{k}\right\}_{k \geq 0}\right)$ is a compact subset of $\mathcal{S}$.

- (SA4) w.p.1, $\lim _{n \rightarrow \infty} \sum_{k=0}^{n} \gamma_{k} e_{k}$ exists and is finite.

Then, w.p.1, $\lim \sup d\left(s_{k}, \mathcal{L}\right)=0$.

The assumptions (SA0-SA3) don't use the dependence structure of the sequence $\left(z_{k}\right)$ and are checked under (M1-M5) (SAEM1-SAEM2) like in the case where the missing data are exactly simulated under the a posteriori distribution (see [5]). Under the assumption (SAEM3), the condition (SA4) is checked because the sequence of the partial sum of $\sum \gamma_{k} e_{k}$ is a convergent martingale. For checking (SA4) under (SAEM3'), we use a result presented in Proposition 7, page 228 of [1]. The general model of the algorithm considered by Benveniste $e t$ al. is of the form:

$$
s_{k}=s_{k-1}+\gamma_{k} H\left(s_{k-1}, z_{k}\right),
$$

where the sequence $\left(s_{k}\right)_{k \geq 0}$ evolves in $\mathbb{R}^{m}$ and the sequence $\left(z_{k}\right)_{k \geq 0}$ in $\mathbb{R}^{l}$. In our algorithm, the function $H$ is equal to:

where the observation $y$ are considered as constant.

$$
H(s, z)=\tilde{S}(y, z)-s,
$$

Proposition (Benveniste et al. [1]). Assume the following assumptions:

- (A1) $\left(\gamma_{k}\right)_{k \geq 0}$ is a decreasing sequence of positive real numbers such that $\sum \gamma_{k}=+\infty$.

- (A2) There exists a family $\left\{\Pi_{\widehat{\theta}(s)}, s \in \mathbb{R}^{m}\right\}$ of transition probabilities on $\mathbb{R}^{l}$ such that for any Borel subset $A$ of $\mathbb{R}^{l}$, we have

$$
P\left(z_{k} \in A \mid \mathcal{F}_{k-1}\right)=\Pi_{\widehat{\theta}\left(s_{k-1}\right)}\left(z_{k-1}, A\right)
$$


- (A3) For any compact subset $Q$ of $\mathcal{S}$, there exists a constant $C_{1}$ depending on $Q$ such that for all $s$ in $Q$ and all $z$ in $\mathcal{E}$ we have

$$
|H(s, z)| \leq C_{1} \text {. }
$$

- (A4) There exists a function $h$ on $\mathcal{S}$ and for each $s$ in $\mathcal{S}$ a function $\nu_{s}$ on $\mathbb{R}^{l}$ such that

1. $h$ is locally Lipschitz on $\mathcal{S}$ : for all $s$ in $\mathcal{S}$, there exist a neighborhood $\mathcal{V}$ of $s$ and a real constant $C$ such that

$$
\forall\left(s^{\prime}, s^{\prime \prime}\right) \in \mathcal{V}^{2} \quad\left|h\left(s^{\prime}\right)-h\left(s^{\prime \prime}\right)\right| \leq C\left|s^{\prime}-s^{\prime \prime}\right| .
$$

2. $\left(I-\Pi_{\widehat{\theta}(s)}\right) \nu_{s}=H_{s}-h(s)$ for all $s$ in $\mathcal{S}$, where for all $z$ in $\mathcal{E}, \Pi_{\widehat{\theta}(s)} \nu_{s}(z) \triangleq \int \nu_{s}\left(z^{\prime}\right) \Pi_{\widehat{\theta}(s)}\left(z, \mathrm{~d} z^{\prime}\right)$.

3. For any compact subset $Q$ of $\mathcal{S}$, there exist real constants $C_{2}, C_{3}$ and $\lambda$ in $\left.] \frac{1}{2}, 1\right]$, such that for all $s$ and $s^{\prime}$ in $Q$ and for all $z$ in $\mathcal{E}$

$$
\begin{aligned}
\left|\nu_{s}(z)\right| & \leq C_{2} \\
\left|\Pi_{\widehat{\theta}(s)} \nu_{s}(z)-\Pi_{\widehat{\theta}\left(s^{\prime}\right)} \nu_{s^{\prime}}(z)\right| & \leq C_{3}\left|s-s^{\prime}\right|^{\lambda} .
\end{aligned}
$$

- (A5) For any compact subset $Q$ of $\mathcal{S}$ and any positive real $q$, there exists a real number $\mu_{q}(Q)$ such that, for all $n$, for all $z_{0}$ in $\mathbb{R}^{l}$ and all $s_{0}$ in $\mathbb{R}^{m}$

$$
E_{z_{0}, s_{0}}\left(\left(1+\left|z_{k}\right|^{q}\right) 11\left(s_{k-1} \in Q, k \leq n\right)\right) \leq \mu_{q}(Q)
$$

where $E_{z_{0}, s_{0}}$ denotes the expectation under the distribution of $\left(z_{k}, s_{k}\right)_{k \geq 0}$ for the initial conditions $\left(z_{0}, s_{0}\right)$.

Then, for any compact subset $Q$ of $\mathcal{S}$, denoting $\tau(Q)=\inf \left\{k, s_{k} \notin Q\right\}$, if the constant $\lambda$ from (A4) verifies $\sum \gamma_{k}^{1+\lambda}<+\infty$, then, on $\{\tau(Q)=\infty\}$, the series $\sum_{k} \gamma_{k} e_{k}$ converges a.s. and in $L^{2}$.

Remark. The proposition of Benveniste et al. requires $\sum \gamma_{k}^{1+\lambda} \leq 1$, but we only need $\sum \gamma_{k}^{1+\lambda}<+\infty$ to obtain the convergence of the series $\sum_{k} \gamma_{k} e_{k}$.

In order to apply this proposition in our case, we have to check the assumptions (A1-A5):

- (A1) is implied by (SAEM1).

- (A2) is verified with the transition probability $\Pi_{\widehat{\theta}(s)}$.

- (A3) is verified since $\tilde{S}$ is bounded on $\mathcal{E}$.

- (A5) is verified since the sequence $\left(z_{k}\right)_{k \geq 0}$ takes its values in a compact subset $\mathcal{E}$ of $\mathbb{R}^{l}$.

Consider now the mean field of the algorithm defined by:

$$
h(s) \triangleq \bar{s}(\widehat{\theta}(s))-s
$$

We will show that this function $h$ satisfies (A4). The assumptions (M3) and (M5) imply that the function $h$ is continuously differentiable on $\mathcal{S}$. So $h$ is locally Lipschitz on $\mathcal{S}$ and assumption (A4) 1 is checked.

We define:

$$
\nu_{s}(z)=\sum_{k \geq 0} \Pi_{\widehat{\theta}(s)}^{k}(H(s, z)-h(s))=\sum_{k \geq 0}\left(\Pi_{\widehat{\theta}(s)}^{k} \tilde{S}(y, z)-p_{\widehat{\theta}(s)} \tilde{S}\right)
$$

where $p_{\widehat{\theta}(s)} \tilde{S} \triangleq \int \tilde{S}(y, z) p_{\widehat{\theta}(s)}(z \mid y) \mathrm{d} z$.

Since $\Pi_{\hat{\theta}(s)}$ is uniformly ergodic, there exist $K_{\hat{\theta}(s)} \in \mathbb{R}^{+}$and $\left.\rho_{\hat{\theta}(s)} \in\right] 0,1[$, such that, for any $k \in \mathbb{N}$, for any $z$ in $\mathcal{E}$

$$
\sup _{\|u\| \leq 1}\left|\Pi_{\widehat{\theta}(s)}^{k} u(z)-p_{\widehat{\theta}(s)} u\right| \leq K_{\hat{\theta}(s)} \rho_{\hat{\theta}(s)}^{k} .
$$


Since $\tilde{S}$ is bounded on $\mathcal{E}$, the series defining $\nu_{s}$ is convergent. Moreover, we have:

$$
\left(I-\Pi_{\widehat{\theta}(s)}\right) \nu_{s}=\tilde{S}-p_{\widehat{\theta}(s)} \tilde{S}
$$

which proves (A4)2.

Under assumption (SAEM3')3 and since $\tilde{S}$ is bounded on $\mathcal{E}$, we obtain the first inequality of (A4)3. We will now prove the second inequality of (A4)3:

$$
\begin{aligned}
\Pi_{\widehat{\theta}(s)} \nu_{s}(z)-\Pi_{\widehat{\theta}\left(s^{\prime}\right)} \nu_{s^{\prime}}(z) & =\sum_{k \geq 1}\left(\Pi_{\widehat{\theta}(s)}^{k} \tilde{S}(y, z)-p_{\widehat{\theta}(s)} \tilde{S}\right)-\sum_{k \geq 1}\left(\Pi_{\widehat{\theta}\left(s^{\prime}\right)}^{k} \tilde{S}(y, z)-p_{\widehat{\theta}\left(s^{\prime}\right)} \tilde{S}\right) \\
& =\nu_{s}(z)-\nu_{s^{\prime}}(z)+p_{\widehat{\theta}(s)} \tilde{S}-p_{\widehat{\theta}\left(s^{\prime}\right)} \tilde{S}
\end{aligned}
$$

so we have

$$
\left|\Pi_{\widehat{\theta}(s)} \nu_{s}(z)-\Pi_{\widehat{\theta}\left(s^{\prime}\right)} \nu_{s^{\prime}}(z)\right| \leq\left|\nu_{s}(z)-\nu_{s^{\prime}}(z)\right|+\left|p_{\widehat{\theta}(s)} \tilde{S}-p_{\widehat{\theta}\left(s^{\prime}\right)} \tilde{S}\right|
$$

We use the following technical lemma (the proof is in the appendix) to prove the second inequality of (A4)3:

Lemma 1. If we assume (SAEM2), (SAEM3') and (C), then for any compact subset $\mathcal{Q}$ of $\mathcal{S}$, there exist $K_{1}$ and $K_{2}$ in $\mathbb{R}^{+}$such that, for any $\left.\alpha \in\right] 0,1\left[\right.$, for any $\left(s, s^{\prime}, z\right) \in \mathcal{Q}^{2} \times \mathcal{E}$,

$$
\left|p_{\widehat{\theta}(s)} \tilde{S}-p_{\widehat{\theta}\left(s^{\prime}\right)} \tilde{S}\right| \leq K_{1}\left|s-s^{\prime}\right|^{\alpha} \quad \text { and } \quad\left|\nu_{s}(z)-\nu_{s^{\prime}}(z)\right| \leq K_{2}\left|s-s^{\prime}\right|^{\alpha}
$$

So we obtain finally that for any compact subset $\mathcal{Q}$ of $\mathcal{S}$, there exists $K$ in $\mathbb{R}^{+}$such that, for any $\left(s, s^{\prime}, z\right) \in \mathcal{Q}^{2} \times \mathcal{E}$,

$$
\left|\Pi_{\widehat{\theta}(s)} \nu_{s}(z)-\Pi_{\widehat{\theta}\left(s^{\prime}\right)} \nu_{s^{\prime}}(z)\right| \leq K\left|s-s^{\prime}\right|
$$

which proves the second inequality of (A4)3 (with $\lambda$ equal to 1 ).

So Proposition 7 of [1] can be applied to prove (SA4). Thus the assumptions of Theorem 2 of [5] are satisfied. The continuity of $\widehat{\theta}: \mathcal{S} \rightarrow \Theta$ and Lemma 2 of [5] prove the convergence of the sequence of estimates $\left(\theta_{k}\right)_{k \geq 0}$.

\section{Applications}

\subsection{The deconvolution problem}

In a convolution model, the observation $\mathbf{y}=\left(y_{L+1}, \ldots, y_{n}\right)$ is the linear convolution of an unobserved input sequence $\mathbf{z}=\left(z_{1}, \ldots, z_{n}\right)$ with additive noise $\varepsilon$, e.g.,

$$
y_{t}=\sum_{l=0}^{L} \varphi_{l} z_{t-l}+\sigma \varepsilon_{t}, \quad L+1 \leq t \leq n
$$

where $\varphi=\left(\varphi_{0}, \ldots, \varphi_{L}\right)$ is the convolution filter.

This kind of model is commonly used in seismic deconvolution, fMRI data analysis and statistical signal analysis.

We shall make the following assumptions, concerning the random sequences $\mathbf{z}$ and $\varepsilon$ : (i) $\left(z_{t}, 1 \leq t \leq n\right)$ is a sequence of independent and identically-distributed random variables, distributed according to some distribution function $\pi$, and taking their values in some compact subset of $\mathbb{R}$. (ii) $\left(\varepsilon_{t}, L+1 \leq t \leq n\right)$ is a sequence of independent standardized Gaussian variables; (iii) $\left(z_{t}, t=1, \cdots, n\right)$ and $\left(\varepsilon_{t}, t=L+1, \cdots, n\right)$ are independent collections of random variables. 
These assumptions together with equation (12) specify completely the log-likelihood of the observed data samples. Let $\mathcal{M}(\mathbf{z})$ be the $(n-L) \times(L+1)$ matrix such that $\mathcal{M}_{i j}(\mathbf{z})=z_{L+1+i-j}$. Then, the complete log-likelihood is, up to a constant,

$$
\log f(\mathbf{y}, \mathbf{z} ; \theta)=-\frac{n-L}{2} \log \sigma^{2}-\frac{1}{2 \sigma^{2}}\|\mathbf{y}-\mathcal{M}(\mathbf{z}) \varphi\|^{2}+\log \pi(\mathbf{z})
$$

Deconvolution consists in recovering the input sequence $\mathbf{z}$ from the observation $\mathbf{y}$. Of course, deconvolution requires an accurate estimation of the convolution filter $\varphi$ and the noise variance $\sigma^{2}$. SAEM will be very useful for estimating these parameters. Furthermore, the marginal distribution $\pi$ of the input sequence $\mathbf{z}$ can also be estimated, whenever it belongs to the exponential family and depends on an unknown parameter $\psi$ :

$$
\pi(\mathbf{z} ; \psi)=C(\psi) \exp \left\{-\left\langle\tilde{S}_{\pi}(\mathbf{z}), \psi\right\rangle\right\}
$$

Here, the vector of parameters of the model is $\theta=\left(\psi, \varphi, \sigma^{2}\right)$ and the minimal sufficient statistics are $\tilde{S}(\mathbf{y}, \mathbf{z})=$ $\left(\mathcal{M}(\mathbf{z})^{t} \mathcal{M}(\mathbf{z}), \mathcal{M}(\mathbf{z})^{t} \mathbf{y}, \tilde{S}_{\pi}(\mathbf{z})\right)$. At step $k$, we used the following procedure for generating $\mathbf{z}_{k}$ from $\mathbf{z}_{k-1}$, using $\theta_{k}=\left(\psi_{k}, \varphi_{k}, \sigma_{k}^{2}\right)$ :

i) a permutation $p_{k}$ of $\{1,2, \ldots, n\}$ is randomly chosen;

ii) for $i=1,2, \ldots, n$ :

1. Let $j=p_{k}(i)$. Set $\tilde{z}_{t}=z_{k-1, t}$ for any $t \neq j$ and generate $\tilde{z}_{j} \sim \pi\left(\cdot ; \psi_{k}\right)$.

2. Compute

$$
\alpha=\frac{1}{2 \sigma_{k}^{2}}\left(\left\|\mathbf{y}-\mathcal{M}(\tilde{\mathbf{z}}) \varphi_{k}\right\|^{2}-\left\|\mathbf{y}-\mathcal{M}(\mathbf{z}) \varphi_{k}\right\|^{2}\right)
$$

3. Generate $u \sim \operatorname{Exp}(1)$. Set $\mathbf{z}_{k}=\tilde{\mathbf{z}}$ if $\alpha<u$ and $\mathbf{z}_{k}=\mathbf{z}_{k-1}$ otherwise.

We can easily show that $\Pi_{\theta_{k}}\left(\mathbf{z}_{k-1}, \cdot\right)$ is the transition probability of an ergodic Markov chain that converges uniformly to the conditional distribution $p\left(\cdot \mid \mathbf{y} ; \theta_{k}\right)$. For estimating $\left(\psi, \boldsymbol{\varphi}, \sigma^{2}\right)$, we define a sequence $\left(s_{k, 1}, s_{k, 2}, s_{k, 3}\right)$ according to (4):

$$
\begin{aligned}
& s_{k, 1}=s_{k-1,1}+\gamma_{k}\left(\mathcal{M}\left(\mathbf{z}_{k}\right)^{t} \mathcal{M}\left(\mathbf{z}_{k}\right)-s_{k-1,1}\right) \\
& s_{k, 2}=s_{k-1,2}+\gamma_{k}\left(\mathcal{M}\left(\mathbf{z}_{k}\right)^{t} \mathbf{y}-s_{k-1,2}\right) \\
& s_{k, 3}=s_{k-1,3}+\gamma_{k}\left(\tilde{S}_{\pi}\left(\mathbf{z}_{k}\right)-s_{k-1,3}\right) .
\end{aligned}
$$

Then, the maximization step yields

$$
\begin{aligned}
\boldsymbol{\varphi}_{k+1} & =\left(s_{k, 1}\right)^{-1} s_{k, 2} \\
\sigma_{k+1}^{2} & =\frac{1}{n-L}\left(\mathbf{y}^{t} \mathbf{y}-\left(s_{k, 2}\right)^{t} \boldsymbol{\varphi}_{k+1}\right) \\
\psi_{k} & =\operatorname{Arg} \max \mathrm{C}(\psi) \mathrm{e}^{-\left\langle\mathrm{s}_{\mathrm{k}, 3}, \psi\right\rangle} .
\end{aligned}
$$

All the assumptions of Theorem 1 are satisfied whenever $\pi$ has bounded support, and the SAEM algorithm converges to a stationary point of the observed likelihood. To assume that the input variables $\mathbf{z}$ are bounded is not a restrictive assumption from a practical point of view. Indeed, any nonbounded distribution is truncated in practice.

We used the convolution model described in (12) for simulating an observed series $\mathbf{y}$ of length $n=500$. In this example, the input sequence $\mathbf{z}$ are independent $\operatorname{Bet} a(a, b)$ random variables on $[0,1]$ with $a=b=3$. So the statistic $\tilde{S}_{\pi}(\mathbf{z})$ is $\left(S_{1}(\mathbf{z}), S_{2}(\mathbf{z})\right)=\left(\sum \log \left(z_{j}\right), \sum \log \left(1-z_{j}\right)\right)$. The convolution filter is $\varphi=(1,-3,2,6,2,-3,1)$. We choose $\sigma^{2}=0.2286$ in order to ensure a Signal to Noise ratio equal to $10 \mathrm{~dB}$, i.e. $\operatorname{Var}(\mathcal{M}(\mathbf{z}) \varphi)=10 \operatorname{Var}(\sigma \varepsilon)$.

Table 1 gives the estimation of $\theta=\left(a, b, \varphi, \sigma^{2}\right)$. A Monte-Carlo experiment based on 100 replications was used for estimating the mean and the standard deviation of two estimators. First, the maximum likelihood 
TABLE 1. Estimation of $\theta=\left(a, b, \boldsymbol{\varphi}, \sigma^{2}\right): \theta^{\star}$ is the true value of $\theta, \theta_{0}$ is the initialization, $\widehat{\theta_{f}}$ is the estimation obtained by maximizing $f(\mathbf{y}, \mathbf{z} ; \theta)$ and $\widehat{\theta_{g}}$ is the estimation obtained by maximizing $g(\mathbf{y} ; \theta)$.

\begin{tabular}{|c|c||c||c|c||c|c|}
\cline { 2 - 7 } \multicolumn{1}{c||}{} & $\theta^{\star}$ & $\theta_{0}$ & $\widehat{\theta_{f}}$ & $\operatorname{std} \widehat{\theta_{f}}$ & $\widehat{\theta_{g}}$ & $\operatorname{std} \widehat{\theta_{g}}$ \\
\hline$a$ & 3 & 0 & 3.0075 & 0.1846 & 2.8615 & 0.4014 \\
$b$ & 3 & 0 & 3.0220 & 0.1838 & 2.6396 & 0.3712 \\
\hline$\varphi_{1}$ & 1 & 0 & 1.0002 & 0.1020 & 1.0277 & 0.5820 \\
$\varphi_{2}$ & -3 & 0 & -3.0058 & 0.0997 & -2.6564 & 0.4612 \\
$\varphi_{3}$ & 2 & 0 & 2.0100 & 0.1046 & 1.8809 & 0.8416 \\
$\varphi_{4}$ & 6 & 1 & 5.9810 & 0.0963 & 5.5637 & 0.4024 \\
$\varphi_{5}$ & 2 & 0 & 2.0034 & 0.1128 & 1.8963 & 0.7713 \\
$\varphi_{6}$ & -3 & 0 & -2.9985 & 0.0955 & -2.7920 & 0.5766 \\
$\varphi_{7}$ & 1 & 0 & 1.0009 & 0.1047 & 0.8361 & 0.5523 \\
\hline$\sigma^{2}$ & 0.2286 & 1 & 0.2266 & 0.0157 & 0.2615 & 0.0593 \\
\hline
\end{tabular}

estimator $\hat{\theta}_{f}$, which maximizes the complete likelihood $f(\mathbf{y}, \mathbf{z} ; \theta)$ assuming that the input series $\mathbf{z}$ is known, was computed as follows:

$$
\begin{aligned}
\hat{\varphi}_{f} & =\left(\mathcal{M}(\mathbf{z})^{t} \mathcal{M}(\mathbf{z})\right)^{-1} \mathcal{M}(\mathbf{z})^{t} \mathbf{y} \\
\hat{\sigma}_{f}^{2} & =\frac{1}{n-L}\left(\mathbf{y}^{t} \mathbf{y}-\mathbf{y}^{t} \mathcal{M}(\mathbf{z}) \hat{\varphi}_{f}\right) \\
\left(\hat{a}_{f}, \hat{b}_{f}\right) & =\operatorname{Arg} \max _{a, b} \frac{1}{B(a, b)} \mathrm{e}^{-(a-1) S_{1}(\mathbf{z})-(b-1) S_{2}(\mathbf{z})},
\end{aligned}
$$

where $B(a, b)=\Gamma(a) \Gamma(b) / \Gamma(a+b)$ and $\Gamma(a)=\int_{0}^{+\infty} \mathrm{e}^{-x} x^{a-1} \mathrm{~d} x$.

On the other hand, the estimator $\hat{\theta}_{g}$ maximizes the incomplete likelihood $g(\mathbf{y} ; \theta)$, considering that the input series $\mathbf{z}$ is unknown.

We computed $\widehat{\theta_{g}}$ with 100 iterations of SAEM, using $\gamma_{k}=1$ for $1 \leq k \leq 30$ and $\gamma_{k}=1 /(k-29)$ for $k \geq 31$.

The initialization is the uniform distribution for $\mathbf{z}\left(a_{0}=b_{0}=0\right)$. The initial guess for the filter is a spike located at $j=4$. That ensures that the algorithm recovers the good phase of the convolution filter. For a different initialization, the algorithm can converge to a local maximum of the likelihood that cannot be compared with the true filter $\varphi^{\star}$. For example, using as initial guess a spike at $j=2$, a simulation gives $\hat{\varphi}=(0.73,6.65,1.42,-3.51,-0.58,2.54,-1.32)$. We remark that the phase of the true filter is not recovered, but the estimated filter and the true filter have both almost the same transfer function. The problem of convergence to the global maximum of the likelihood is beyond the scope of this paper (see [11] for a simulated annealing version of this algorithm).

The results presented in Table 1 confirm that $\widehat{\theta_{f}}$ is a more accurate estimate of $\theta$ than $\widehat{\theta_{g}}$. Nevertheless, we can remark that, when $\mathbf{z}$ is not observed, SAEM provides a good estimation of $\theta$.

The approximation scheme presented Section 2.3 for estimating the covariance of the estimate $\widehat{\theta_{g}}$ requires to compute the derivative and the second derivative of the complete log-likelihood. Unfortunately, the estimation of the covariance of the estimate of $(a, b)$ is very intricate in this example, since the derivative of the Gamma function cannot be computed in a close-form. For this reason, we will only consider the estimation of the covariance of the estimated filter $\hat{\varphi}$. The other parameters are assumed to be known in this experiment. 
TABLE 2. Comparison of the Monte-Carlo variance $\operatorname{Var}_{M C}\left(\widehat{\theta_{g}}\right)$ with the Monte-Carlo asymptotic variance $\overline{\operatorname{Var}_{F i}\left(\widehat{\theta_{g}}\right)}$. The initialization $\theta_{0}$ is the true value $\theta^{\star}$.

\begin{tabular}{|c||c|c||c|c|}
\cline { 2 - 5 } \multicolumn{1}{c||}{} & $\theta^{\star}$ & $\theta_{0}$ & $\operatorname{Var}_{M C}\left(\widehat{\theta_{g}}\right)$ & $\overline{\operatorname{Var}_{F i}\left(\widehat{\theta_{g}}\right)}$ \\
\hline$\varphi_{1}$ & 1 & 1 & 0.041 & 0.016 \\
$\varphi_{2}$ & -3 & -3 & 0.027 & 0.016 \\
$\varphi_{3}$ & 2 & 2 & 0.018 & 0.016 \\
$\varphi_{4}$ & 6 & 6 & 0.033 & 0.015 \\
$\varphi_{5}$ & 2 & 2 & 0.019 & 0.016 \\
$\varphi_{6}$ & -3 & -3 & 0.033 & 0.016 \\
$\varphi_{7}$ & 1 & 1 & 0.022 & 0.016 \\
\hline
\end{tabular}

We have used the SAEM algorithm with the 100 simulated series for estimating the convolution filter $\varphi$. The covariance of the estimate was estimated by the Monte-Carlo covariance $\operatorname{Var}_{M C}\left(\widehat{\theta_{g}}\right)$ defined by:

$$
\operatorname{Var}_{M C}\left(\widehat{\theta_{g}}\right)=\frac{1}{100} \sum_{i=1}\left({\widehat{\theta_{g}}}^{(i)}-\theta^{\star}\right)\left({\widehat{\theta_{g}}}^{(i)}-\theta^{\star}\right)^{\prime}
$$

where ${\widehat{\theta_{g}}}^{(i)}$ is the estimate of $\theta^{\star}$ obtained with the $i$ th simulated data set.

We also estimated the asymptotic covariance $I_{g}\left(\theta^{\star}\right)^{-1}$ using the procedure presented Section 2.3. The MonteCarlo asymptotic covariance $\overline{\operatorname{Var}_{F i}\left(\widehat{\theta_{g}}\right)}$ is defined by:

$$
\overline{\operatorname{Var}_{F i}\left(\widehat{\theta_{g}}\right)}=\frac{1}{100} \sum_{i=1} \operatorname{Var}_{F i}\left({\widehat{\theta_{g}}}^{(i)}\right)
$$

where $\operatorname{Var}_{F i}\left(\widehat{\theta}_{g}^{(i)}\right)$ is the estimate of asymptotic covariance obtained with the $i$ th simulated data set.

The diagonal elements of both estimates $\operatorname{Var}_{M C}\left(\widehat{\theta_{g}}\right)$ and $\overline{\operatorname{Var}_{F i}\left(\widehat{\theta_{g}}\right)}$ are displayed Table 2. We can make the following comments about these results:

- The estimate of the asymptotic variance fluctuates very few. Indeed, the mean value of each diagonal component of the series $\left(\operatorname{Var}_{F i}\left(\widehat{\theta}_{g}^{(i)}\right) ; 1 \leq i \leq 100\right)$ is around 0.016 , with a standard deviation of only 0.001. Then we can expect that the asymptotic covariance is well estimated with only one observed series using this procedure.

- The two estimates do not match very closely, but a confidence interval can be obtained with the SAEM procedure. Indeed, the asymptotic standard deviations are around 0.13 , while the standard deviations of the SAEM estimate are between 0.14 and 0.20 .

- The Monte-Carlo estimated variances are bigger than the asymptotic variances. Several explanations can be proposed. First, the Central Limit Theorem proposed in (7) is an asymptotic result that does not necessarily apply for a series of length $n=500$. On the other hand, according to the initial guess the SAEM algorithm can converge to any local maxima of the observed likelihood instead of the MLE. Thus, if the likelihood function is very rife, with several local maxima, the variance of the SAEM estimate can be bigger than the variance of the MLE. 


\subsection{The change-points problem}

We use the model considered in [10]. We observe a real sequence $\mathbf{y}=\left(y_{i}, 1 \leq i \leq n\right)$, such that, for any $1 \leq i \leq n$,

$$
y_{i}=f\left(t_{i}\right)+\sigma \varepsilon_{i}
$$

where $\left(t_{i}, 1 \leq i \leq n\right)$ is a sequence of known observation times and $\left(\varepsilon_{i}, 1 \leq i \leq n\right)$ is a sequence of independent zero-mean Gaussian variables with unit variance. The function $f$ to recover is piecewise constant. Thus, there exists a sequence of instants $\left(\tau_{j}, j \geq 0\right)$ among the sequence $\left(t_{i}, 1 \leq i \leq n\right)$ and a sequence $\left(m_{j}, j \geq 1\right)$ such that, for any $j \geq 1$,

$$
f(t)=m_{j} \text { for all } \tau_{j-1}<t \leq \tau_{j},
$$

with the convention $\tau_{0}=0$.

We introduce a latent sequence of independent identically-distributed Bernoulli random variables $\left(z_{i}, 1 \leq\right.$ $i \leq n-1)$ that take the value 1 at the change instants and 0 between two changes:

$$
z_{i}=\left\{\begin{array}{l}
1 \text { if there exists } j \text { such that } t_{i}=\tau_{j} \\
0 \text { otherwise. }
\end{array}\right.
$$

Let $\lambda$ be the parameter of the Bernoulli and for any $\mathbf{z}=\left(z_{i}, 1 \leq i \leq n-1\right)$ in $\Omega=\{0,1\}^{n-1}$, let $K_{\mathbf{z}}=\sum_{i=1}^{n-1} z_{i}+1$ be the number of segments (i.e. $K_{\mathbf{z}}-1$ is the number of change-points) defined by $\mathbf{z}$. Then,

$$
\pi(\mathbf{z} ; \lambda)=\lambda^{K_{\mathbf{z}}-1}(1-\lambda)^{n-K_{\mathbf{z}}} .
$$

Conditionally to the change-points sequence, the vector $\mathbf{m}=\left(m_{j}, 1 \leq j \leq K_{\mathbf{z}}\right)$ is assumed to be Gaussian:

$$
\pi(\mathbf{m} \mid \mathbf{z} ; \mu, V)=\prod_{j=1}^{K_{\mathbf{z}}}\left(\frac{2 \pi V}{n_{j}}\right)^{-\frac{1}{2}} \exp \left\{-\frac{n_{j}}{2 V}\left(m_{j}-\mu\right)^{2}\right\}
$$

where $n_{j}=\sum_{i=1}^{n} 1_{\left.]_{j-1}, \tau_{j}\right]}\left(t_{i}\right)$ is the number of observations in segment $] \tau_{j-1}, \tau_{j}$ ] (where the function $f$ is constant equal to $m_{j}$ ) for $1 \leq j \leq K_{\mathbf{z}}$.

On the other hand, $\left(\varepsilon_{i}, 1 \leq i \leq n\right)$ is assumed to be a sequence of independent zero-mean and unit variance Gaussian random variables. Thus, the conditional distribution of the observations is defined by:

$$
h\left(\mathbf{y} \mid \mathbf{z}, \mathbf{m} ; \sigma^{2}\right)=\left(2 \pi \sigma^{2}\right)^{-\frac{n}{2}} \exp \left\{-\frac{1}{2 \sigma^{2}} \sum_{j=1}^{K_{\mathbf{z}}} \sum_{l=N_{j-1}+1}^{N_{j}}\left(y_{l}-m_{j}\right)^{2}\right\}
$$

where $N_{j}=\sum_{l=1}^{j} n_{l}$ for $1 \leq j \leq K_{\mathbf{z}}$ and $N_{0}=0$.

Remark. If the collection of observation times $\left(t_{i}, 1 \leq i \leq n\right)$ is equal to $(1, \cdots, n)$, then $n_{j}=\tau_{j}-\tau_{j-1}$ and $N_{j}=\tau_{j}$ for $1 \leq j \leq K_{\mathbf{z}}$ and some expressions are quite simplified.

Let $\theta=\left(\mu, \lambda, V, \sigma^{2}\right)$ be the set of hyperparameters of the model. For any configuration of changes $\mathbf{z}$, let $\bar{y}_{j}=n_{j}^{-1} \sum_{l=N_{j-1}+1}^{N_{j}} y_{l}, \bar{y}=n^{-1} \sum_{l=1}^{n} y_{l}$ and $C_{\mathbf{z}}=\sum_{j=1}^{K_{\mathbf{z}}} \sum_{l=N_{j-1}+1}^{N_{j}}\left(y_{l}-\bar{y}_{j}\right)^{2}$. Then, after some calculation, it can be shown (see [10]) that the likelihood of $(\mathbf{y}, \mathbf{z})$ is defined by

$$
f(\mathbf{y}, \mathbf{z} ; \theta)=\left(2 \pi \sigma^{2}\right)^{-\frac{n}{2}}\left(\frac{\sigma^{2}+V}{\sigma^{2}}\right)^{-\frac{K_{\mathbf{z}}}{2}} \lambda^{K_{\mathbf{z}}-1}(1-\lambda)^{n-K_{\mathbf{z}}} \exp \left\{-\frac{1}{2\left(V+\sigma^{2}\right)}\left(\sum_{i=1}^{n}\left(y_{i}-\mu\right)^{2}+\frac{V}{\sigma^{2}} C_{\mathbf{z}}\right)\right\} .
$$


TABLE 3. Estimation of $\theta=\left(\lambda, V, \sigma^{2}\right): \theta^{\star}$ is the true value of $\theta, \theta_{0}$ is the initialization, $\hat{\theta}_{f}$ is the estimation obtained by maximizing $f(\mathbf{y}, \mathbf{z} ; \theta), \hat{\theta}_{g}$ is the estimation obtained by maximizing $g(\mathbf{y} ; \theta), \operatorname{Var}_{M C}\left(\widehat{\theta_{g}}\right)$ is the Monte-Carlo variance and $\overline{\operatorname{Var}_{F i}\left(\widehat{\theta_{g}}\right)}$ is the Monte-Carlo asymptotic variance.

\begin{tabular}{|c|c||c||c|c||c|c||c|c|}
\cline { 2 - 9 } \multicolumn{1}{c||}{} & $\theta^{\star}$ & $\theta_{0}$ & $\widehat{\widehat{\theta_{f}}}$ & $\operatorname{std} \widehat{\operatorname{\theta _{f}}}$ & $\widehat{\widehat{\theta_{g}}}$ & $\operatorname{std} \widehat{\theta_{g}}$ & $\operatorname{Var}_{M C}\left(\widehat{\theta_{g}}\right)$ & $\overline{\operatorname{Var}_{F i}\left(\widehat{\theta_{g}}\right)}$ \\
\hline$\lambda$ & 0.02 & 0.05 & 0.0201 & 0.0051 & 0.0235 & 0.0105 & $12.2 \times 10^{-5}$ & $5.6 \times 10^{-5}$ \\
\hline$V$ & 40 & 10 & 38.1 & 15.8 & 32.5 & 19.0 & 417.25 & 116.32 \\
\hline$\sigma^{2}$ & 1 & 5 & 1.01 & 0.07 & 1.01 & 0.08 & 0.0065 & 0.0042 \\
\hline
\end{tabular}

Here, the minimal sufficient statistics $\left(K_{\mathbf{z}}, C_{\mathbf{z}}\right)$ are approximated according to (4):

$$
\begin{aligned}
& s_{k, 1}=s_{k-1,1}+\gamma_{k}\left(K_{\mathbf{z}}-s_{k-1,1}\right) \\
& s_{k, 2}=s_{k-1,2}+\gamma_{k}\left(C_{\mathbf{z}}-s_{k-1,2}\right) .
\end{aligned}
$$

Then, the maximization step yields

$$
\begin{aligned}
\mu_{k} & =\bar{y} \\
\lambda_{k} & =\frac{s_{k, 1}-1}{n-1} \\
\sigma_{k}^{2} & =\frac{s_{k, 2}}{n-s_{k, 1}} \\
V_{k} & =\frac{\sum_{i=1}^{n}\left(y_{i}-\bar{y}\right)^{2}-s_{k, 2}}{s_{k, 1}}-\sigma_{k}^{2} .
\end{aligned}
$$

Here, $\mathbf{z}$ takes a finite number of values. Then, any irreducible proposal kernel $q$ can be used to generate a geometrically ergodic kernel. In this application, we used alternatively the three following kernels: $i$ ) a new vector $\tilde{\mathbf{z}}$ is drawn independently of the current value $\mathbf{z}_{k-1}$ with the marginal distribution $\pi\left(\cdot ; \psi_{k-1}\right)$; ii) a new change-point is created, or an existing change-point is removed; iii) an existing change-point is shifted (see [10] for more details concerning the MCMC procedure).

The maximum likelihood estimate of $\mu$ is $\bar{y}$. For estimating the other hyperparameters, the SAEM algorithm can be used since it is easy to check that the assumptions of Theorem 1 are still satisfied.

We used the change-point model described above for simulating an observed series $\mathbf{y}$ of length $n=500$. We set the values of the hyperparameters to $\lambda^{\star}=0.02, V^{\star}=40$ and $\sigma^{2^{\star}}=1$.

Table 3 gives the estimation of $\theta=\left(\lambda, V, \sigma^{2}\right)$. A Monte-Carlo experiment based on 100 replications was used for estimating the mean and the standard deviation of $\hat{\theta}_{f}$, which maximizes the complete likelihood $f(\mathbf{y}, \mathbf{z} ; \theta)$ and $\hat{\theta}_{g}$ that maximizes the incomplete likelihood $g(\mathbf{y} ; \theta)$. We computed $\hat{\theta}_{g}$ with 100 iterations of SAEM, using $\gamma_{k}=1$ for $1 \leq k \leq 30$ and $\gamma_{k}=1 /(k-29)$ for $k \geq 31$.

In this example, SAEM still produces a good estimation of $\theta$. In particular, the noise variance $\sigma^{2}$ is estimated with almost the same accuracy, when the change-points are known and when they are unknown.

The Monte-Carlo variance $\operatorname{Var}_{M C}\left(\widehat{\theta_{g}}\right)$ and the Monte-Carlo asymptotic variance $\overline{\operatorname{Var}_{F i}\left(\widehat{\theta_{g}}\right)}$ are also displayed Table 3. We can make the same remarks as in the previous example: the variance of the SAEM estimate is bigger than the asymptotic variance. Nevertheless, a confidence interval can be obtained using the SAEM algorithm. 


\section{A. Appendix}

We first prove a technical lemma which will be necessary to prove Lemma 1.

Lemma 2. If we assume (SAEM2), (SAEM3') and (C), then for any compact subset $\mathcal{Q}$ of $\mathcal{S}$, there exists a real constant $M$ such that:

$$
\forall\left(s, s^{\prime}, y, z\right) \in \mathcal{Q}^{2} \times \mathcal{Y} \times \mathcal{E} \quad \forall k \in \mathbb{N} \quad\left|\Pi_{\widehat{\theta}(s)}^{k} \tilde{S}(y, z)-\Pi_{\widehat{\theta}\left(s^{\prime}\right)}^{k} \tilde{S}(y, z)\right| \leq M k\left|s-s^{\prime}\right| .
$$

Proof. $\forall\left(s, s^{\prime}\right) \in \mathcal{Q}^{2} \quad \forall(y, z) \in \mathcal{Y} \times \mathcal{E} \quad \forall k \in \mathbb{N}$,

$$
\begin{aligned}
\left|\Pi_{\widehat{\theta}(s)}^{k} \tilde{S}(y, z)-\Pi_{\widehat{\theta}\left(s^{\prime}\right)}^{k} \tilde{S}(y, z)\right| & \leq \sum_{i=0}^{k-1}\left|\Pi_{\widehat{\theta}(s)}^{k-i} \Pi_{\widehat{\theta}\left(s^{\prime}\right)}^{i} \tilde{S}(y, z)-\Pi_{\widehat{\theta}(s)}^{k-1-i} \Pi_{\widehat{\theta}\left(s^{\prime}\right)}^{i+1} \tilde{S}(y, z)\right| \\
& =\sum_{i=0}^{k-1}\left|\Pi_{\widehat{\theta}(s)}^{k-1-i}\left(\Pi_{\widehat{\theta}(s)}-\Pi_{\widehat{\theta}\left(s^{\prime}\right)}\right) \Pi_{\widehat{\theta}\left(s^{\prime}\right)}^{i} \tilde{S}(y, z)\right| \\
& =\sum_{i=0}^{k-1}\left|\iiint \Pi_{\widehat{\theta}\left(s^{\prime}\right)}^{i}(z, u)\left(\Pi_{\widehat{\theta}(s)}(u, v)-\Pi_{\widehat{\theta}\left(s^{\prime}\right)}(u, v)\right) \Pi_{\widehat{\theta}(s)}^{k-1-i}(v, w) \tilde{S}(y, w) \mathrm{d} u \mathrm{~d} v \mathrm{~d} w\right| \\
& \leq\|\tilde{S}\|_{\infty} \sum_{i=0}^{k-1} \iiint \Pi_{\widehat{\theta}\left(s^{\prime}\right)}^{i}(z, u)\left|\Pi_{\widehat{\theta}(s)}(u, v)-\Pi_{\widehat{\theta}\left(s^{\prime}\right)}(u, v)\right| \Pi_{\widehat{\theta}(s)}^{k-1-i}(v, w) \mathrm{d} u \mathrm{~d} v \mathrm{~d} w .
\end{aligned}
$$

The assumption (SAEM2) ensures that the set $\hat{\theta}(\mathcal{Q})$ is compact, so that the assumption (SAEM3')2 ensures the existence of real constants $L$ and $\tilde{L}$ such that:

$$
\begin{aligned}
\left|\Pi_{\widehat{\theta}(s)}^{k} \tilde{S}(y, z)-\Pi_{\widehat{\theta}\left(s^{\prime}\right)}^{k} \tilde{S}(y, z)\right| & \leq\|\tilde{S}\|_{\infty} L\left|\hat{\theta}(s)-\hat{\theta}\left(s^{\prime}\right)\right| \sum_{i=0}^{k-1} \iiint \Pi_{\widehat{\theta}\left(s^{\prime}\right)}^{i}(z, u) \Pi_{\widehat{\theta}(s)}^{k-1-i}(v, w) \mathrm{d} u \mathrm{~d} v \mathrm{~d} w \\
& \leq\|\tilde{S}\|_{\infty} \tilde{L}\left|s-s^{\prime}\right| \sum_{i=0}^{k-1} \iiint \Pi_{\widehat{\theta}\left(s^{\prime}\right)}^{i}(z, u) \Pi_{\widehat{\theta}(s)}^{k-1-i}(v, w) \mathrm{d} u \mathrm{~d} v \mathrm{~d} w
\end{aligned}
$$

since $\hat{\theta}$ is continuously differentiable. Moreover $\mathcal{E}$ is compact, so we obtain:

$$
\left|\Pi_{\widehat{\theta}(s)}^{k} \tilde{S}(y, z)-\Pi_{\widehat{\theta}\left(s^{\prime}\right)}^{k} \tilde{S}(y, z)\right| \leq\|\tilde{S}\|_{\infty} \tilde{L} \mathrm{M}(\mathcal{E}) k\left|s-s^{\prime}\right|,
$$

where $\mathrm{M}(A)$ denotes the Lebesgue measure of the set $A$.

Proof of Lemma 1. Assumptions (SAEM3')1 and (SAEM3')3 imply the existence of constants $K \in \mathbb{R}^{+}$ and $\rho \in] 0,1\left[\operatorname{such}\right.$ that for all $\left(s, s^{\prime}\right)$ in $\mathcal{Q}^{2}$, for all $(y, z)$ in $\mathcal{Y} \times \mathcal{E}$ and for all $k$ in $\mathbb{N}$, we have

$$
\begin{aligned}
\left|p_{\widehat{\theta}(s)} \tilde{S}-p_{\widehat{\theta}\left(s^{\prime}\right)} \tilde{S}\right| & \leq\left|p_{\widehat{\theta}(s)} \tilde{S}-\Pi_{\widehat{\theta}(s)}^{k} \tilde{S}(y, z)\right|+\left|\Pi_{\widehat{\theta}(s)}^{k} \tilde{S}(y, z)-\Pi_{\widehat{\theta}\left(s^{\prime}\right)}^{k} \tilde{S}(y, z)\right|+\left|\Pi_{\widehat{\theta}\left(s^{\prime}\right)}^{k} \tilde{S}(y, z)-p_{\widehat{\theta}\left(s^{\prime}\right)} \tilde{S}\right| \\
& \leq 2 \|\left.\tilde{S}\right|_{\infty} K \rho^{k}+M k\left|s-s^{\prime}\right| .
\end{aligned}
$$

We choose $k \approx \log \left(\left|s-s^{\prime}\right|\right) / \log (\rho)$ so that the two terms have approximatively the same weight. Then, there exist two constants $K_{1}$ in $\mathbb{R}^{+}$and $c$ in $] 0,1$ [ such that

$$
\forall\left(s, s^{\prime}\right) \in \mathcal{Q}^{2} \quad \text { s.t. } \quad\left|s-s^{\prime}\right|<c, \quad\left|p_{\widehat{\theta}(s)} \tilde{S}-p_{\widehat{\theta}\left(s^{\prime}\right)} \tilde{S}\right| \leq \frac{K_{1}}{\log (\rho)} \log \left(\left|s-s^{\prime}\right|\right)\left|s-s^{\prime}\right| .
$$


Since we have

$$
\forall \alpha \in] 0,1\left[\lim _{\left|s-s^{\prime}\right| \rightarrow 0} \log \left(\left|s-s^{\prime}\right|\right)\left|s-s^{\prime}\right|^{1-\alpha}=0,\right.
$$

we can deduce that $\forall \alpha \in] 0,1[, \exists \eta>0$ s.t.

$$
\forall\left(s, s^{\prime}\right) \in \mathcal{Q}^{2} \quad \text { s.t. } \quad\left|s-s^{\prime}\right|<\eta, \quad\left|p_{\widehat{\theta}(s)} \tilde{S}-p_{\widehat{\theta}\left(s^{\prime}\right)} \tilde{S}\right| \leq K_{1}\left|s-s^{\prime}\right|^{\alpha},
$$

which proves the first inequality of Lemma 1 for all pair $\left(s, s^{\prime}\right)$ in a compact $\mathcal{Q}$ such that $\left|s-s^{\prime}\right|<\eta$.

Concerning the second inequality of Lemma 1, let us define

$$
a_{k, s, s^{\prime}}(y, z)=\left|\left(\Pi_{\widehat{\theta}(s)}^{k} \tilde{S}(y, z)-p_{\widehat{\theta}(s)} \tilde{S}\right)-\left(\Pi_{\widehat{\theta}\left(s^{\prime}\right)}^{k} \tilde{S}(y, z)-p_{\widehat{\theta}\left(s^{\prime}\right)} \tilde{S}\right)\right|
$$

On one hand, since $\tilde{S}$ is bounded and $\Pi_{\widehat{\theta}(s)}$ is uniformly ergodic, there exist $K \in \mathbb{R}^{+}$and $\left.\rho \in\right] 0,1[$ such that, for any $k \in \mathbb{N}$ and any $\left(s, s^{\prime}, y, z\right) \in \mathcal{Q}^{2} \times \mathcal{Y} \times \mathcal{E}$,

$$
\begin{aligned}
a_{k, s, s^{\prime}}(y, z) & \leq\left|\Pi_{\widehat{\theta}(s)}^{k} \tilde{S}(y, z)-p_{\widehat{\theta}(s)} \tilde{S}\right|+\left|\Pi_{\widehat{\theta}\left(s^{\prime}\right)}^{k} \tilde{S}(y, z)-p_{\widehat{\theta}\left(s^{\prime}\right)} \tilde{S}\right| \\
& \leq 2\|\tilde{S}\|_{\infty} K \rho^{k} .
\end{aligned}
$$

On the other hand, for any $\beta \in] 0,1\left[\right.$, there exists $\eta>0$ such that, for any $k \in \mathbb{N}$ and any $\left(s, s^{\prime}, y, z\right) \in \mathcal{Q}^{2} \times \mathcal{Y} \times \mathcal{E}$ such that $\left|s-s^{\prime}\right|<\eta$,

$$
\begin{aligned}
a_{k, s, s^{\prime}}(y, z) & \leq\left|\Pi_{\widehat{\theta}(s)}^{k} \tilde{S}(y, z)-\Pi_{\widehat{\theta}\left(s^{\prime}\right)}^{k} \tilde{S}(y, z)\right|+\left|p_{\widehat{\theta}(s)} \tilde{S}-p_{\widehat{\theta}\left(s^{\prime}\right)} \tilde{S}\right| \\
& \leq M k\left|s-s^{\prime}\right|+K_{2}\left|s-s^{\prime}\right|^{\beta} \leq \max \left(M, K_{2}\right)(k+1)\left|s-s^{\prime}\right|^{\beta} .
\end{aligned}
$$

Then, using a convexity argument, there exist $L_{1} \in \mathbb{R}^{+}, L_{2} \in \mathbb{R}^{+}$and $L \in \mathbb{R}^{+}$such that, for any $\left.a \in\right] 0,1[$, for any $\beta \in] 0,1\left[\right.$, there exists $\eta>0$ such that, for any $k \in \mathbb{N}$ and any $\left(s, s^{\prime}, y, z\right) \in \mathcal{Q}^{2} \times \mathcal{Y} \times \mathcal{E}$ such that $\left|s-s^{\prime}\right|<\eta$,

$$
\begin{aligned}
a_{k, s, s^{\prime}}(y, z) & \leq \min \left(L_{1} \rho^{k}, L_{2}(k+1)\left|s-s^{\prime}\right|^{\beta}\right) \\
& \leq L \rho^{(1-a) k}\left|s-s^{\prime}\right|^{\beta a}(k+1)^{a} .
\end{aligned}
$$

Thus, for any $\beta \in] 0,1[$, for any $a \in] 0,1\left[\right.$, there exists $\eta>0$ such that, for any $\left(s, s^{\prime}, y, z\right) \in \mathcal{Q}^{2} \times \mathcal{Y} \times \mathcal{E}$ such that $\left|s-s^{\prime}\right| \leq \eta$

$$
\begin{aligned}
\left|\nu_{s}(z)-\nu_{s^{\prime}}(z)\right| & \leq \sum_{k \geq 0} a_{k, s, s^{\prime}}(y, z) \\
& \leq L\left(\sum_{k \geq 0} \rho^{(1-a) k}(k+1)^{a}\right)\left|s-s^{\prime}\right|^{a \beta}
\end{aligned}
$$

So we obtain the following conclusion: for any $a \in] 0,1[$, for any $\alpha \in] 0, a\left[\right.$, there exist real constants $K_{2}$ and $\eta$ such that for all $z$ in $\mathcal{E}$

$$
\forall\left(s, s^{\prime}\right) \in \mathcal{Q}^{2} \quad \text { s.t. } \quad\left|s-s^{\prime}\right|<\eta, \quad\left|\nu_{s}(z)-\nu_{s^{\prime}}(z)\right| \leq K_{2}\left|s-s^{\prime}\right|^{\alpha} .
$$

Since $\mathcal{Q}$ is compact, we can recover it with a finite number $N$ of balls of diameter $\eta$ and thus obtain the inequalities (25) and (26) with the same constants multiplied by $N$ for any pair $\left(s, s^{\prime}\right)$ in $\mathcal{Q}^{2}$. 


\section{REFERENCES}

[1] A. Benveniste, M. Métivier and P. Priouret, Adaptive algorithms and stochastic approximations. Springer-Verlag, Berlin (1990). Translated from the French by Stephen S. Wilson.

[2] O. Brandière and M. Duflo, Les algorithmes stochastiques contournent-ils les pièges ? C. R. Acad. Sci. Paris Ser. I Math. 321 (1995) 335-338.

[3] H.F. Chen, G. Lei and A.J. Gao, Convergence and robustness of the Robbins-Monro algorithm truncated at randomly varying bounds. Stochastic Process. Appl. 27 (1988) 217-231.

[4] D. Concordet and O.G. Nunez, A simulated pseudo-maximum likelihood estimator for nonlinear mixed models. Comput. Statist. Data Anal. 39 (2002) 187-201.

[5] B. Delyon, M. Lavielle and E. Moulines, Convergence of a stochastic approximation version of the EM algorithm. Ann. Statist. 27 (1999) 94-128.

[6] A.P. Dempster, N.M. Laird and D.B. Rubin, Maximum likelihood from incomplete data via the EM algorithm. J. R. Stat. Soc. Ser. B 39 (1977) 1-38.

[7] M.G. Gu and F.H. Kong, A stochastic approximation algorithm with Markov chain Monte-Carlo method for incomplete data estimation problems. Proc. Natl. Acad. Sci. USA 95 (1998) 7270-7274 (electronic).

[8] M.G. Gu and H.-T. Zhu, Maximum likelihood estimation for spatial models by Markov chain Monte Carlo stochastic approximation. J. R. Stat. Soc. Ser. B 63 (2001) 339-355.

[9] K. Lange, A gradient algorithm locally equivalent to the EM algorithm. J. R. Stat. Soc. Ser. B 57 (1995) $425-437$.

[10] M. Lavielle and E. Lebarbier, An application of MCMC methods to the multiple change-points problem. Signal Processing $\mathbf{8 1}$ (2001) 39-53.

[11] M. Lavielle and E. Moulines, A simulated annealing version of the EM algorithm for non-Gaussian deconvolution. Statist. Comput. 7 (1997) 229-236.

[12] X.-L. Meng and D.B. Rubin, Maximum likelihood estimation via the ECM algorithm: a general framework. Biometrika 80 (1993) 267-278.

[13] K.L. Mengersen and R.L. Tweedie, Rates of convergence of the Hastings and Metropolis algorithms. Ann. Statist. 24 (1996) $101-121$.

[14] S.P. Meyn and R.L. Tweedie, Markov chains and stochastic stability, Springer-Verlag London Ltd., London. Comm. Control Engrg. Ser. (1993).

[15] C.-F. Jeff Wu, On the convergence properties of the EM algorithm. Ann. Statist. 11 (1983) 95-103.

[16] J.-F. Yao, On recursive estimation in incomplete data models. Statistics 34 (2000) 27-51 (English). 